\title{
On Repeated Values of the Riemann Zeta Function on the Critical Line
}

\author{
William D. Banks and Sarah Kang
}

\section{CONTENTS}

1. Introduction

2. Ordinates of Zeros on the Critical Line

3. Loops

4. Two Criteria for Goodness

5. Self-Intersecting Loops

6. Intersections between Distinct Loops

7. Description of Numerical Methods

8. Concluding Remarks

References

2000 AMS Subject Classification: 11M26, 11M06

Keywords: Riemann zeta function, critical line, repeated values, loops
Let $\zeta(s)$ be the Riemann zeta function. In this paper, we study repeated values of $\zeta(s)$ on the critical line, and we give evidence to support our conjecture that for every nonzero complex number $z$, the equation $\zeta(1 / 2+i t)=z$ has at most two solutions $t \in \mathbb{R}$. We prove a number of related results, some of which are unconditional, and some of which depend on the truth of the Riemann hypothesis. We also propose some related conjectures that are implied by Montgomery's pair correlation conjecture.

\section{INTRODUCTION}

The Riemann zeta function $\zeta(s)$ lies at the heart of analytic number theory. In the half-plane $\{s=\sigma+i t \in \mathbb{C}$ : $\sigma>1\}$ it can be defined either as a Dirichlet series

$$
\zeta(s)=\sum_{n=1}^{\infty} n^{-s}
$$

or (equivalently) as an Euler product

$$
\zeta(s)=\prod_{p \text { prime }}\left(1-p^{-s}\right)^{-1}
$$

In Bernhard Riemann's extraordinary memoir [Riemann 59], it is shown that $\zeta(s)$ extends to a meromorphic function on the whole complex plane with its only singularity being a simple pole at $s=1$ and that it satisfies a functional equation relating its values at $s$ and $1-s$. There are many excellent accounts of the theory of the Riemann zeta function; we refer the reader to [Borwein et al. 08, Edwards 74, Ingham 90, Ivić 85, Karatsuba and Voronin 92, Patterson 88, Titchmarsh 86] and the references contained therein.

In the half-plane $\mathcal{H}=\left\{\sigma>\frac{1}{2}\right\}$, the zeta function $\zeta(s)$ takes every nonzero complex value infinitely often (cf. [Titchmarsh 86, Theorem 11.10]), whereas the Riemann hypothesis $(\mathrm{RH})$ asserts that $\zeta(s) \neq 0$ for every $s \in \mathcal{H}$; in particular, the Riemann hypothesis implies that

$\{z \in \mathbb{C}: \zeta(s)=z$ for infinitely many $s \in \mathcal{H}\}=\mathbb{C} \backslash\{0\}$ 
In March 2008, at the analytic number theory workshop in Oberwolfach, the first author gave empirical evidence that the complementary result

$$
\{z \in \mathbb{C}: \zeta(s)=z \text { for infinitely many } s \in \mathcal{L}\}=\{0\}
$$

is likely to hold on the boundary $\mathcal{L}$ of the half-plane $\mathcal{H}$, that is, on the critical line $\mathcal{L}=\left\{\sigma=\frac{1}{2}\right\}$; this result had been conjectured earlier by Selberg in a footnote to his 1989 paper on Dirichlet series [Selberg 92]. The purpose of the present note is to describe some (albeit limited) numerical evidence that we have obtained in support of Selberg's conjecture (1-1). Moreover, our findings suggest that the following stronger statement may be true.

Conjecture 1.1. For every complex number $z \neq 0$, the equation $\zeta(1 / 2+i t)=z$ has at most two solutions $t \in \mathbb{R}$.

Note that this conjecture implies (1-1) in view of Hardy's famous result [Hardy 14$]$ that $\zeta(s)$ has infinitely many zeros on the critical line.

The analysis of data from our numerical computations has also led us to some unconditional results that show that there are many complex numbers $z \neq 0$ such that $\zeta(1 / 2+i t)=z$ has at least two solutions $t \in \mathbb{R}$ (and thus we expect that there are precisely two solutions for every such $z)$. We shall say that a closed interval $[a, b]$ is good if there exist two infinite sequences of real numbers, $\left(t_{k}\right)_{k=1}^{\infty}$ and $\left(t_{k}^{*}\right)_{k=1}^{\infty}$, such that

(i) $\left(t_{k}\right)_{k=1}^{\infty}$ is contained in $[a, b]$;

(ii) $\left(t_{k}^{*}\right)_{k=1}^{\infty}$ is unbounded;

(iii) $\zeta\left(1 / 2+i t_{k}\right)=\zeta\left(1 / 2+i t_{k}^{*}\right) \neq 0$ for every $k$.

Theorem 1.2. Let $\lambda=3.4362182260 \ldots$ be the least positive real number for which $\zeta(1 / 2+i \lambda) \in \mathbb{R}$. Then the interval $[-\lambda, \lambda]$ is good.

This is proved in Section 5 using a criterion for "goodness" (Lemma 4.1) that is given in Section 4. In a similar spirit, we prove the following statement in Section 6 .

Theorem 1.3. Let $\gamma_{126}=279.2292509277 \ldots$ and $\gamma_{127}=$ $282.4651147650 \ldots$ be the ordinates of the two zeros $\rho=$ $1 / 2+i \gamma$ of $\zeta(s)$ with $279<\gamma<283$. Then the interval $\left[\gamma_{126}, \gamma_{127}\right]$ is good.

In Section 5, we prove a conditional result concerning loops in the graph of $\zeta(1 / 2+i t), t \in \mathbb{R}$. To formulate the theorem, suppose that $\mathrm{RH}$ is true, and let $0<\tau_{1}<$ $\tau_{2}<\cdots$ be the sequence of distinct ordinates of the zeros $\rho=1 / 2+i \gamma$ of $\zeta(s)$ with $\gamma>0$. For each $n \geqslant 1$, the loop $\mathscr{L}_{n}$ is the collection of complex numbers given by

$$
\mathscr{L}_{n}=\left\{\zeta(1 / 2+i t): \tau_{n}<t<\tau_{n+1}\right\}
$$

(see Section 3 for a more general definition of $\mathscr{L}_{n}$ that does not require the assumption of $\mathrm{RH}$ ). Note that $0 \notin$ $\mathscr{L}_{n}$, but $\mathscr{L}_{n} \cup\{0\}$ is a closed curve.

Theorem 1.4. Assume RH. Then there are infinitely many $n$ such that $\mathscr{L}_{n}$ does not intersect itself, and there are infinitely many $n$ for which $\mathscr{L}_{n}$ has a self-intersection.

Corollary 1.5. Assume RH. Then for every $\varepsilon>0$, there are real numbers $t_{1} \neq t_{2}$ with $\left|t_{1}-t_{2}\right|<\varepsilon$ and $\zeta(1 / 2+$ $\left.i t_{1}\right)=\zeta\left(1 / 2+i t_{2}\right) \neq 0$.

Corollary 1.6. Assume RH. Then for every $\varepsilon>0$, there exists a good interval $[a, b]$ of length $b-a<\varepsilon$.

We also propose the following conjecture, which may follow from the truth of $\mathrm{RH}$ and Montgomery's pair correlation conjecture via a variant of Theorem 1.4.

Conjecture 1.7. For every $k \geqslant 1$ there is a loop $\mathscr{L}_{n}$ with $k$ self-intersections.

A related conjecture for pairs of loops is given in Section 6 .

\section{ORDINATES OF ZEROS ON THE CRITICAL LINE}

Let

$$
\cdots<\tau_{-2}<\tau_{-1}<\tau_{0}<\tau_{1}<\tau_{2}<\cdots
$$

be the sequence of distinct real solutions to the equation $\zeta(1 / 2+i t)=0$, arranged in increasing order, with $\tau_{1}=$ $14.1347 \ldots$ being the least positive solution. According to this definition, we have

$$
\zeta(1 / 2+i t) \neq 0 \quad\left(\tau_{n}<t<\tau_{n+1}\right) .
$$

Note that

$$
\tau_{n}=-\tau_{1-n} \quad(n \in \mathbb{Z})
$$

which follows from the fact that $\overline{\zeta(1 / 2+i t)}=\zeta(1 / 2-i t)$ for all $t \in \mathbb{R}$.

As usual, we also arrange the zeros $\beta+i \gamma$ of $\zeta(s)$ with $\gamma>0$ in a sequence $\rho_{n}=\beta_{n}+i \gamma_{n}$ so that $\gamma_{n+1} \geqslant \gamma_{n}$. From computations in [Gourdon and Demichel 04], it is 


\begin{tabular}{r|r}
\multicolumn{1}{c|}{$n$} & \multicolumn{1}{c}{$\tau_{n}$} \\
\hline-5 & $-37.58617815882567125721 \ldots$ \\
-4 & $-32.93506158773918969066 \ldots$ \\
-3 & $-30.42487612585951321031 \ldots$ \\
-2 & $-25.01085758014568876321 \ldots$ \\
-1 & $-21.02203963877155499262 \ldots$ \\
0 & $-14.13472514173469379045 \ldots$ \\
1 & $14.13472514173469379045 \ldots$ \\
2 & $21.02203963877155499262 \ldots$ \\
3 & $25.01085758014568876321 \ldots$ \\
4 & $30.42487612585951321031 \ldots$ \\
5 & $32.93506158773918969066 \ldots$
\end{tabular}

TABLE 1. Ordinates of zeros on the critical line.

known that $\beta_{n}=1 / 2$ and $\gamma_{n+1}>\gamma_{n}$ for all natural numbers $n \leqslant 10^{13}$; hence $\tau_{n}=\gamma_{n}$ for every such $n$. By $(2-2)$, we also have $\tau_{n}=-\gamma_{1-n}$ in the range $-10^{13}<n \leqslant 0$. Thus for small values of $|n|$, the number $\tau_{n}$ can be evaluated with arbitrary numerical precision using, for instance, the function ZetaZero in Mathematica. Table 1 gives values of $\tau_{n}$ with $-5 \leqslant n \leqslant 5$ :

\section{LOOPS}

For every $n \in \mathbb{Z}$ we define the loop $\mathscr{L}_{n}$ to be the collection of complex numbers given by

$$
\mathscr{L}_{n}=\left\{\zeta(1 / 2+i t): \tau_{n}<t<\tau_{n+1}\right\} .
$$

In view of $(2-2)$, it follows that $\overline{\mathscr{L}_{n}}=\mathscr{L}_{-n}$ for all $n \in \mathbb{Z}$. Also, by $(2-1)$, we see that zero is not contained in any set $\mathscr{L}_{n}$; hence $\bigcup_{n \in \mathbb{Z}} \mathscr{L}_{n}$ is the complete set of nonzero values taken by $\zeta(s)$ on the critical line.

\section{TWO CRITERIA FOR GOODNESS}

Lemma 4.1. Let $a, b \in \mathbb{R}$ with $a<b$ and $\zeta(1 / 2+i a)=$ $\zeta(1 / 2+i b)$. Suppose that

$$
\mathscr{C}=\{\zeta(1 / 2+i t): a \leqslant t \leqslant b\}
$$

is a Jordan curve in $\mathbb{C}$ that encloses an open neighborhood of zero. Then the interval $[a, b]$ is good.

Proof. Let $M=\max _{t \in[a, b]}|\zeta(1 / 2+i t)|$. Since $\zeta(s)$ is unbounded on the critical line, there is a sequence $\left(t_{k}^{\circ}\right)_{k=1}^{\infty}$ such that $\left|\zeta\left(1 / 2+i t_{k}^{\circ}\right)\right|>M+k$ for all $k$. Let $n_{k}$ be the integer for which $\tau_{n_{k}}<t_{k}^{\circ}<\tau_{n_{k}+1}$; clearly, the sequence $\left(n_{k}\right)_{k=1}^{\infty}$ is unbounded. For each $k$, since $\zeta\left(1 / 2+i \tau_{n_{k}}\right)=$ 0 lies inside the curve $\mathscr{C}$ and $\zeta\left(1 / 2+i t_{k}^{\circ}\right)$ lies outside, there is a real number $t_{k}^{*}$ in the range $\tau_{n_{k}}<t_{k}^{*}<t_{k}^{\circ}$

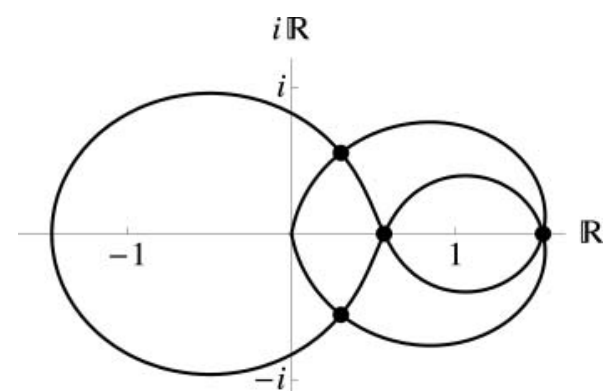

FIGURE 1. The loop $\mathscr{L}_{0}$.

such that $\zeta\left(1 / 2+i t_{k}^{*}\right)$ lies on the curve $\mathscr{C}$; that is, $\zeta\left(1 / 2+i t_{k}^{*}\right)=\zeta\left(1 / 2+i t_{k}\right) \neq 0$ for some $t_{k} \in[a, b]$. Since the sequence $\left(n_{k}\right)_{k=1}^{\infty}$ is unbounded, the same is true of $\left(t_{k}^{*}\right)_{k=1}^{\infty}$, and hence the sequences $\left(t_{k}\right)_{k=1}^{\infty}$ and $\left(t_{k}^{*}\right)_{k=1}^{\infty}$ satisfy the conditions (i), (ii), and (iii) in Section 1.

With a slight modification to the above proof, the interval $[a, b]$ in Lemma 4.1 can be replaced by any finite union of closed intervals.

Lemma 4.2. Let $\mathcal{U}$ be a finite union of closed intervals in $\mathbb{R}$, and suppose that the set

$$
\mathscr{C}=\{\zeta(1 / 2+i t): t \in \mathcal{U}\}
$$

is a Jordan curve in $\mathbb{C}$ that encloses an open neighborhood of zero. Then $\mathscr{C} \cap \mathscr{L}_{n} \neq \varnothing$ for infinitely many $n \in \mathbb{Z}$.

\section{SELF-INTERSECTING LOOPS}

Proof of Theorem 1.2. The loop $\mathscr{L}_{0}$ (see Figure 1) has four self-intersections, which are given in Table 2 .

If $\lambda=3.4362 \ldots$ is the least positive real number such that $\zeta(1 / 2+i \lambda) \in \mathbb{R}$, then $\mathscr{C}=\{\zeta(1 / 2+i t):-\lambda \leqslant t \leqslant$ $\lambda\}$ is a Jordan curve in $\mathbb{C}$ that encloses an open neighborhood of zero, as seen in Figure 2. Applying Lemma 4.1, we immediately obtain the statement of Theorem 1.2.

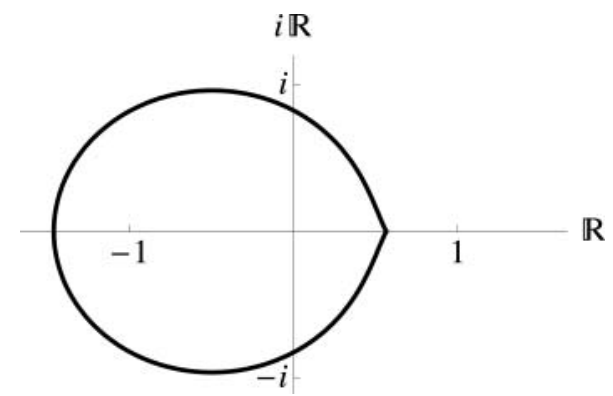

FIGURE 2. The Jordan curve $\mathscr{C}=\{\zeta(1 / 2+i t):-\lambda \leqslant$ $t \leqslant \lambda\}$. 


\begin{tabular}{c|c|l}
$t_{1}$ & $t_{2}$ & \multicolumn{1}{|c}{$\zeta\left(1 / 2+i t_{1}\right)$ and $\zeta\left(1 / 2+i t_{2}\right)$} \\
\hline$-13.26322741 \ldots$ & $-1.33231317 \ldots$ & $0.30051216 \ldots+i \cdot 0.55357158 \ldots$ \\
$-9.66690805 \ldots$ & $9.66690805 \ldots$ & $1.53182067 \ldots$ \\
$-3.43621822 \ldots$ & $3.43621822 \ldots$ & $0.56415097 \ldots$ \\
$13.26322741 \ldots$ & $1.33231317 \ldots$ & $0.30051216 \ldots-i \cdot 0.55357158 \ldots$
\end{tabular}

TABLE 2. Self-intersections of $\mathscr{L}_{0}$.

In our numerical investigation, we were originally concerned only with intersections between distinct loops $\mathscr{L}_{m} \neq \mathscr{L}_{n}$ (see Section 6 below). However, to have a complete understanding of the repeated values of $\zeta(s)$ on the critical line, one must also consider loops with self-intersections. Initially, we did not expect to find selfintersecting loops other than the loop $\mathscr{L}_{0}$; however, in studying the question, we were led to Theorem 1.4, which suggests the existence of infinitely many such loops. Using a specialized search, we subsequently found the selfintersecting loop $\mathscr{L}_{379}$, which is shown in Figure 3.

Proof of Theorem 1.4. As usual, if $t$ is not the ordinate of a zero of the zeta function, we define $\arg \zeta(s)=\Im \log \zeta(s)$ by continuous variation from $\infty+i t$ to $\sigma+i t$, and if $t>$ 0 , we denote by $N(t)$ the number of zeros $\rho=\beta+i \gamma$ of $\zeta(s)$ in the rectangle $0<\beta<1,0<\gamma<t$. To simplify the notation somewhat, we put $\vartheta(t)=\arg \zeta(1 / 2+i t)$ for any such $t$.

Let $b>a \geqslant 1$ and suppose that the closed interval $[a, b]$ does not contain the ordinate of a zero of $\zeta(s)$. We begin with the well-known identity (cf. [Montgomery and Vaughan 07, Theorem 14.1])

$$
\vartheta(t)=\pi(N(t)-1)-\arg \Gamma(1 / 4+i t / 2)+\frac{t}{2} \log \pi .
$$

Since $N(t)$ is constant on $[a, b]$, it follows that

$$
\vartheta^{\prime}(t)=-\frac{1}{2} \Re \frac{\Gamma^{\prime}}{\Gamma}(1 / 4+i t / 2)+\frac{1}{2} \log \pi \quad(t \in[a, b]) .
$$

Using the estimate (cf. [Montgomery and Vaughan 07, Theorem C.1])

$$
\frac{\Gamma^{\prime}}{\Gamma}(s)=\log s+O(1 /|s|)
$$

in the special case that $s=1 / 4+i t / 2$, we derive that

$$
\vartheta^{\prime}(t)=-\frac{1}{2} \log t+\frac{1}{2} \log 2 \pi+O\left(t^{-1}\right) \quad(t \in[a, b]),
$$

which in turn yields the estimate

$$
\vartheta(b)-\vartheta(a)=-\frac{1}{2}(b-a) \log a+O(b-a) .
$$

Note that the implied constants in (5-1) and (5-2) are absolute.
Assuming RH, we can take $a \rightarrow \gamma_{n}^{+}$and $b \rightarrow \gamma_{n+1}^{-}$in the discussion above. From (5-2), we see that

$$
\theta_{1, n}-\theta_{2, n}=\frac{1}{2}\left(\gamma_{n+1}-\gamma_{n}\right) \log \gamma_{n}+o(1) \quad(n \rightarrow \infty)
$$

where

$$
\theta_{1, n}=\lim _{a \rightarrow \gamma_{n}^{+}} \vartheta(a) \text { and } \quad \theta_{2, n}=\lim _{b \rightarrow \gamma_{n+1}^{-}} \vartheta(b) .
$$

It is shown in [Montgomery 73] that (under $\mathrm{RH}$ ) the number of simple zeros $\rho=1 / 2+i \gamma$ of $\zeta(s)$ with $0<$ $\gamma \leqslant T$ is not less than $(2 / 3+o(1)) N(T)$; from this it follows that there are at least $(3+o(1)) N(T)$ simple zeros with $T<\gamma \leqslant 6 T$. Since there are at least as many gaps between zeros as there are simple zeros, it follows that there are infinitely many $n$ such that

$$
0<\gamma_{n+1}-\gamma_{n}<\frac{5}{3} \cdot \frac{2 \pi}{\log \gamma_{n}} .
$$

For any sufficiently large $n$ with this property, combining (5-3) and (5-4), we deduce that

$$
0<\theta_{1, n}-\theta_{2, n}<2 \pi
$$

and (5-1) implies that $\vartheta(t)$ is strictly decreasing on the interval $\left(\gamma_{n}, \gamma_{n+1}\right)$; consequently, the map

$$
t \mapsto e^{i \vartheta(t)}=\frac{\zeta(1 / 2+i t)}{|\zeta(1 / 2+i t)|}
$$

is injective on $\left(\gamma_{n}, \gamma_{n+1}\right)$, i.e., the loop $\mathscr{L}_{n}$ does not intersect itself.

In the other direction, it is shown in [Conrey et al. 84] that the truth of $\mathrm{RH}$ implies that

$$
\lim \sup \left(\gamma^{\prime}-\gamma\right) \frac{\log \gamma}{2 \pi}>2.337
$$

where $\gamma \leqslant \gamma^{\prime}$ are consecutive ordinates of zeros of $\zeta(s)$. Hence, under $\mathrm{RH}$ there are infinitely many $n$ such that

$$
\gamma_{n+1}-\gamma_{n}>\frac{7}{3} \cdot \frac{2 \pi}{\log \gamma_{n}}
$$

If $n$ has this property and is large enough, then combining (5-3) and (5-5), we deduce that

$$
\theta_{1, n}-\theta_{2, n}>2 \pi
$$




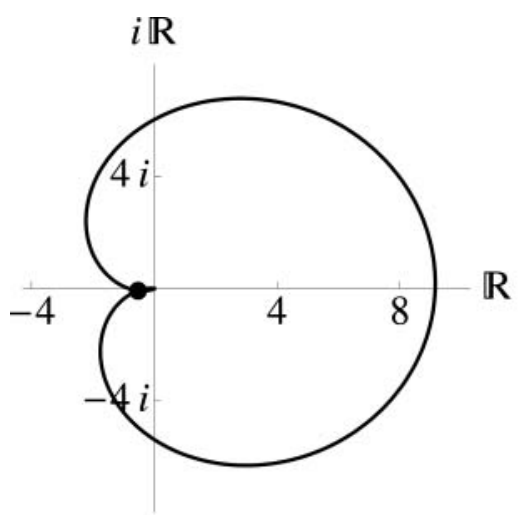

(a)

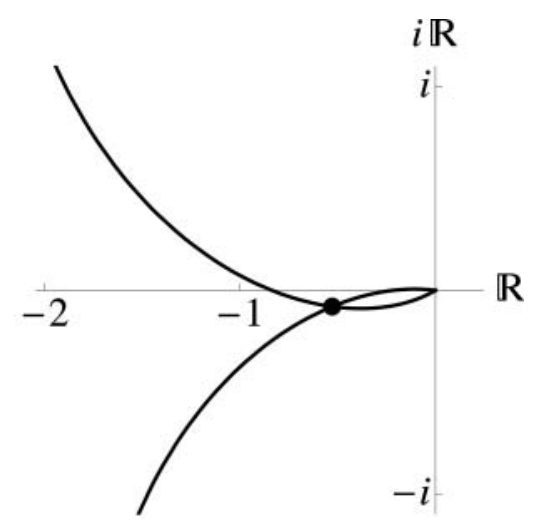

(b)

FIGURE 3. The self-intersecting loop $\mathscr{L}_{379}$.

As before, using (5-1), we see that $\vartheta(t)$ is strictly decreasing on the interval $\left(\gamma_{n}, \gamma_{n+1}\right)$ for large $n$; thus the map $\vartheta:\left(\gamma_{n}, \gamma_{n+1}\right) \rightarrow\left(\theta_{2, n}, \theta_{1, n}\right)$ is invertible; let $\vartheta^{-1}$ : $\left(\theta_{2, n}, \theta_{1, n}\right) \rightarrow\left(\gamma_{n}, \gamma_{n+1}\right)$ be the inverse map. Let

$$
f(\theta)=\left|\zeta\left(1 / 2+i \vartheta^{-1}(\theta)\right)\right|-\left|\zeta\left(1 / 2+i \vartheta^{-1}(\theta+2 \pi)\right)\right|
$$

for $\theta_{2, n}<\theta<\theta_{1, n}-2 \pi$.

Since

$$
f\left(\theta_{2, n}^{+}\right)=-\left|\zeta\left(1 / 2+i \vartheta^{-1}\left(\theta_{2, n}+2 \pi\right)\right)\right|<0
$$

and

$$
f\left(\theta_{1, n}^{-}-2 \pi\right)=\left|\zeta\left(1 / 2+i \vartheta^{-1}\left(\theta_{1, n}-2 \pi\right)\right)\right|>0,
$$

there is a number $\theta \in\left(\theta_{2, n}, \theta_{1, n}-2 \pi\right)$ such that $f(\theta)=0$; that is,

$$
\left|\zeta\left(1 / 2+i t_{1}\right)\right|=\left|\zeta\left(1 / 2+i t_{2}\right)\right|
$$

where

$$
t_{1}=\vartheta^{-1}(\theta) \quad \text { and } \quad t_{2}=\vartheta^{-1}(\theta+2 \pi) .
$$

Since

$$
\arg \zeta\left(1 / 2+i t_{1}\right)=\theta \quad \text { and } \quad \arg \zeta\left(1 / 2+i t_{2}\right)=\theta+2 \pi,
$$

it follows that

$$
\zeta\left(1 / 2+i t_{1}\right)=\zeta\left(1 / 2+i t_{2}\right) .
$$

Since $t_{1}$ and $t_{2}$ are distinct elements in $\left(\gamma_{n}, \gamma_{n+1}\right)$, this shows that $\mathscr{L}_{n}$ has a self-intersection.

Theorem 1.4 shows (under $\mathrm{RH}$ ) that there are infinitely many $n$ with the property that there are real numbers $t_{1}, t_{2} \in\left(\gamma_{n}, \gamma_{n+1}\right), t_{1} \neq t_{2}$, such that $\zeta(1 / 2+$ $\left.i t_{1}\right)=\zeta\left(1 / 2+i t_{2}\right) \neq 0$; for example, take $t_{1}$ and $t_{2}$ as defined in (5-6). Since the length $\gamma_{n+1}-\gamma_{n}$ of the interval $\left(\gamma_{n}, \gamma_{n+1}\right)$ tends to zero as $n \rightarrow \infty$, Corollary 1.5 follows.
To obtain Corollary 1.6, we apply Lemma 4.1 with the Jordan curve $\mathscr{C}=\left\{\zeta(1 / 2+i t): t_{1} \leqslant t \leqslant t_{2}\right\}$.

\section{INTERSECTIONS BETWEEN DISTINCT LOOPS}

In this section, we discuss our results about intersections between distinct loops $\mathscr{L}_{m} \neq \mathscr{L}_{n}$. Figure 4(a) illustrates the fairly typical situation in which a loop pair $\left(\mathscr{L}_{m}, \mathscr{L}_{n}\right)$ has a single intersection. We also found many loop pairs with no intersections; this is illustrated in Figure 4(b), which graphs the loops $\mathscr{L}_{n}$ with $n \in$ $\{-64,-43,1,2,3,4,8,16,33,53,55\}$, no two of which intersect.

Table 3 discloses, for various values of $N$, the total number of loop pairs $\left(\mathscr{L}_{m}, \mathscr{L}_{n}\right)$ with $-N \leqslant m<n \leqslant N$, the overall number of intersections that are found among such pairs, and the number of such pairs having precisely $0,1,2,3,4$, or 5 intersections.

We did not encounter any loop pairs with more than five intersections in our limited investigation. Nevertheless, we propose the following conjecture, which is related to Conjecture 1.7 .

Conjecture 6.1. Let $\iota_{k}(N)$ be the number of pairs $(m, n)$, $-N \leqslant m<n \leqslant N$, such that the loops $\mathscr{L}_{m}$ and $\mathscr{L}_{n}$ have precisely $k$ intersections. Then there are constants $N_{0}(k)$ and $c_{k}>0$ such that $\iota_{k}(N) \geqslant c_{k} N$ for all $N \geqslant N_{0}(k)$.

The primary aim of our numerical experiment was to gather evidence in support of Conjecture 1.1. It is easy to see that if $z \neq 0$ and the equation $\zeta(1 / 2+i t)=z$ has more than two solutions $t \in \mathbb{R}$, then at least one of the following possibilities must occur: 


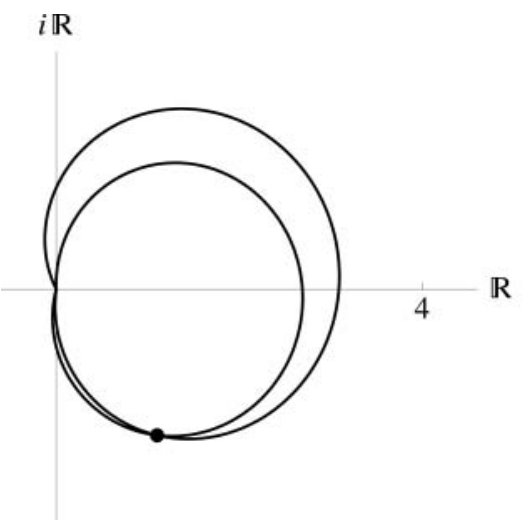

(a)

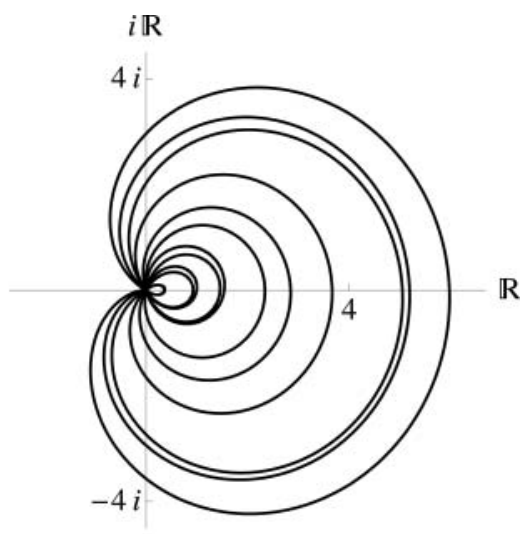

(b)

FIGURE 4. Intersecting and nonintersecting loops.

(i) there is a loop that intersects itself three times at the same point;

(ii) there is a loop that has a self-intersection at a point that also lies on another loop;

(iii) there is a point that lies on three distinct loops.

Our focus was on the loops $\mathscr{L}_{n}$ with $|n| \leqslant 100$, and we did not encounter any loops satisfying (i). To eliminate the possibilities (ii) and (iii) within our loop set, we located and precisely evaluated 8933 points of intersection: the four self-intersections on loop $\mathscr{L}_{0}$ together with an additional 8929 intersections between distinct loops $\mathscr{L}_{m} \neq \mathscr{L}_{n}$ (see Section 7 for a description of our methods). We found no instance of a point satisfying either (ii) or (iii). Of the 8933 intersection points we considered, the closest pair is separated by a distance exceeding $5.28687 \times 10^{-7}$, and this pair occurs where $\mathscr{L}_{-19}$ intersects $\mathscr{L}_{39}$ and $\mathscr{L}_{100}$ (see Figure 5). Table 4 gives information about the relevant points of intersection.

Since $\overline{\mathscr{L}_{n}}=\mathscr{L}_{-n}$ for every $n \in \mathbb{Z}$, the loops $\mathscr{L}_{n}$ and $\mathscr{L}_{-n}$ have a real point of intersection whenever $\mathscr{L}_{n}$ crosses the real axis; these were first studied in [Gram 03] and are called Gram points. Of the 8933 intersection points considered, only the Gram points were found to lie on the real axis, although some points of intersection lie quite close to the real axis (see Figure 6). We propose the following conjecture, which is a consequence of Conjecture 1.1.

Conjecture 6.2. If $\mathscr{L}_{m}$ and $\mathscr{L}_{n}$ are distinct loops with a point of intersection on the real axis, then $m=-n$.

To finish this section, let us now turn to the proof of Theorem 1.3.

Proof of Theorem 1.3. We first observe that the loops $\mathscr{L}_{126}$ and $\mathscr{L}_{-126}$ have two intersections, which are given in Table 5.

Let $\lambda_{1}=280.8024 \ldots$ and $\lambda_{2}=282.4547 \ldots$ be the two real numbers $t_{1}$ in the open interval $\left(\gamma_{126}, \gamma_{127}\right)$ such that $\zeta\left(1 / 2+i t_{1}\right) \in \mathbb{R}$. If $\mathcal{U}=\left[\lambda_{1}, \lambda_{2}\right] \cup\left[-\lambda_{2},-\lambda_{1}\right]$, then one verifies that $\mathscr{C}=\{\zeta(1 / 2+i t): t \in \mathcal{U}\}$ is a Jordan curve in $\mathbb{C}$ that encloses an open neighborhood of zero.

\begin{tabular}{c|c|c|c|c|c|c|c|c}
$N$ & \# pairs & total ints. & 0 int. & 1 int. & 2 int. & 3 int. & 4 int. & 5 int. \\
\hline 10 & 210 & 108 & 142 & 48 & 8 & 4 & 8 & 0 \\
20 & 820 & 378 & 530 & 248 & 18 & 8 & 10 & 6 \\
30 & 1830 & 784 & 1176 & 588 & 34 & 10 & 12 & 10 \\
40 & 3240 & 1377 & 2045 & 1097 & 56 & 14 & 14 & 14 \\
50 & 5050 & 2147 & 3143 & 1778 & 74 & 17 & 20 & 18 \\
60 & 7260 & 3127 & 4437 & 2648 & 110 & 21 & 24 & 20 \\
70 & 9870 & 4270 & 5976 & 3675 & 144 & 21 & 26 & 28 \\
80 & 12880 & 5571 & 7769 & 4840 & 182 & 25 & 28 & 36 \\
90 & 16290 & 7297 & 9551 & 6406 & 230 & 27 & 30 & 46 \\
100 & 20100 & 8929 & 11823 & 7874 & 290 & 29 & 32 & 52
\end{tabular}

TABLE 3. Data on intersections between loop pairs $\left(\mathscr{L}_{m}, \mathscr{L}_{n}\right.$ with- $N \leq m<n \leq N$. 


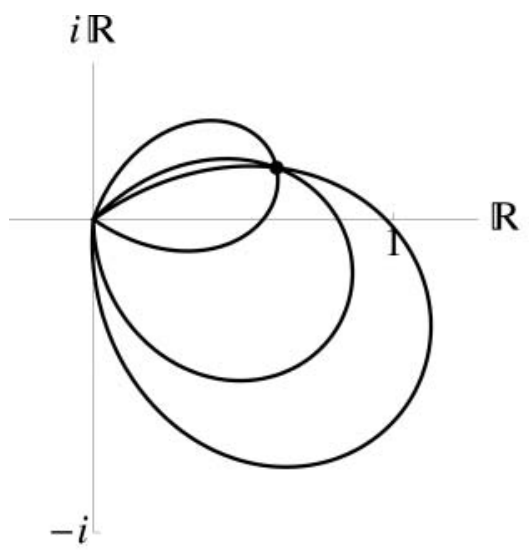

(a)

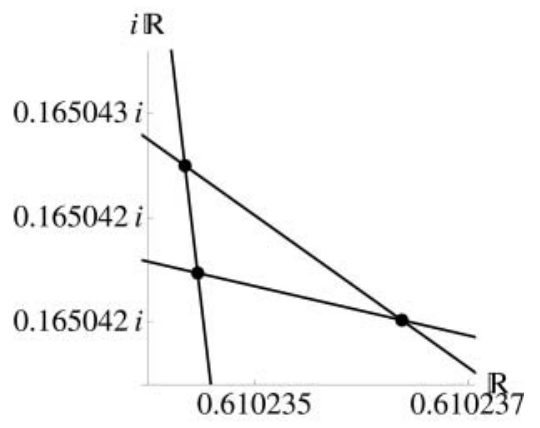

(b)

FIGURE 5. Intersections of the loops $\mathscr{L}_{-19}, \mathscr{L}_{39}$, and $\mathscr{L}_{100}$.

Applying Lemma 4.2 , we see that $\mathscr{C} \cap \mathscr{L}_{n} \neq \varnothing$ for infinitely many $n \in \mathbb{Z}$. Since $\mathscr{C}$ is a subset of $\mathscr{L}_{126} \cup \mathscr{L}_{-126}$, it follows that one of the following two cases occurs for infinitely many $n$ :

(i) $\mathscr{L}_{126} \cap \mathscr{L}_{n} \neq \varnothing$;

(ii) $\mathscr{L}_{-126} \cap \mathscr{L}_{n} \neq \varnothing$.

However, since $\mathscr{L}_{126} \cap \mathscr{L}_{n} \neq \varnothing$ if and only if $\mathscr{L}_{-126} \cap$ $\mathscr{L}_{-n} \neq \varnothing$, both cases (i) and (ii) must occur for infinitely many $n$, which finishes the proof of Theorem 1.3.

\section{DESCRIPTION OF NUMERICAL METHODS}

In this section, we briefly describe our method for computing intersections between loops. Our computations were performed using Mathematica, which we selected for its ease of use, its built-in library, and its display capabilities.

The built-in function FindRoot in Mathematica is exceedingly convenient for numerically evaluating intersections between loops (i.e., repeated values of the zeta function) to any desired level of precision. However, to ensure that no intersections were missed among the more than 20,000 loop pairs under consideration, and in order to automate our use of FindRoot, we first needed to find crude approximations to the locations of the intersections. To do so, our basic data object was an ordered quadruple of real numbers called a quad. Each quad was given in the form of a list $\mathcal{Q}=\left\{t_{1, b}, t_{1, e}, t_{2, b}, t_{2, e}\right\}$, and it represented two arcs on the graph of $\zeta(s)$ defined by

$$
\begin{aligned}
& \mathcal{A}_{1}=\left\{\zeta(1 / 2+i t): t_{1, b}<t<t_{1, e}\right\}, \\
& \mathcal{A}_{2}=\left\{\zeta(1 / 2+i t): t_{2, b}<t<t_{2, e}\right\} .
\end{aligned}
$$

For a given loop pair $\left(\mathscr{L}_{m}, \mathscr{L}_{n}\right)$ with $m \neq n$, we defined an initial quad $\mathcal{Q}$ by taking

$$
\begin{aligned}
& t_{1, b}=\tau_{m}+\delta=\gamma_{m}+\delta, \quad t_{1, e}=\tau_{m+1}-\delta=\gamma_{m+1}-\delta, \\
& t_{2, b}=\tau_{n}+\delta=\gamma_{n}+\delta, \quad t_{2, e}=\tau_{n+1}-\delta=\gamma_{n+1}-\delta,
\end{aligned}
$$

where $\delta>0$ is a predetermined parameter. Note that $\mathcal{A}_{1} \approx \mathscr{L}_{m}$ and $\mathcal{A}_{2} \approx \mathscr{L}_{n}$ when $\delta$ is small. In our computation, the value of $\delta$ was chosen to be small enough that all intersections between the loops $\mathscr{L}_{m}$ and $\mathscr{L}_{n}$ would still be present as intersections between the $\operatorname{arcs} \mathcal{A}_{1}$ and $\mathcal{A}_{2}$. On the other hand, $\delta$ was large enough that the FindRoot command, once it was invoked, would not converge to the value zero, i.e., to the limiting value of $\zeta(s)$ near the boundaries of the intervals $\left(\tau_{m}, \tau_{m+1}\right)$ and $\left(\tau_{n}, \tau_{n+1}\right)$.

Once defined, the initial quad was placed in a list (containing only the one quad). The following cycle was performed twenty times:

(i) Each quad $\mathcal{Q}=\left\{t_{1, b}, t_{1, e}, t_{2, b}, t_{2, e}\right\}$ in the current list was split into four distinct subquads by splitting both

\begin{tabular}{c|c|c|c} 
Loop pair & $t_{1}$ & $t_{2}$ & $\zeta\left(1 / 2+i t_{1}\right)$ and $\zeta\left(1 / 2+i t_{2}\right)$ \\
\hline$\left(\mathscr{L}_{-19}, \mathscr{L}_{39}\right)$ & $-76.38206310 \ldots$ & $121.71273069 \ldots$ & $0.61023434 \ldots+i \cdot 0.16504225 \ldots$ \\
$\left(\mathscr{L}_{-19}, \mathscr{L}_{100}\right)$ & $-76.38206243 \ldots$ & $236.70765230 \ldots$ & $0.61023446 \ldots+i \cdot 0.16504173 \ldots$ \\
$\left(\mathscr{L}_{39}, \mathscr{L}_{100}\right)$ & $121.71273203 \ldots$ & $236.70765293 \ldots$ & $0.61023638 \ldots+i \cdot 0.16504150 \ldots$
\end{tabular}

TABLE 4. Intersections of $\mathscr{L}_{-19}, \mathscr{L}_{39}$ and $\mathscr{L}_{100}$. 


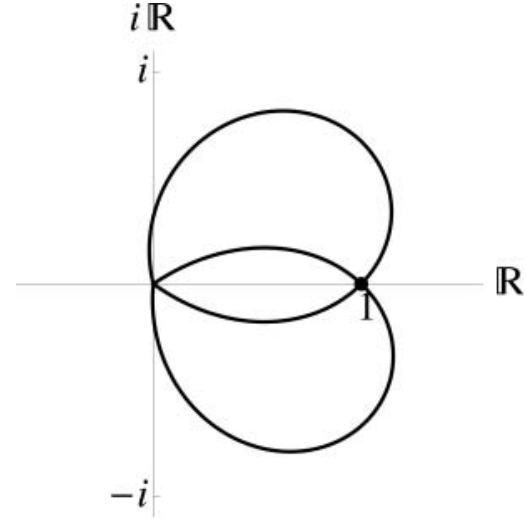

(a)

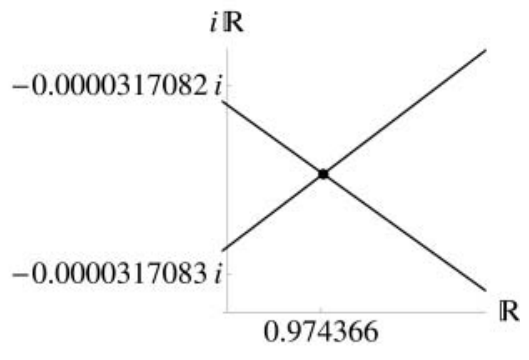

(b)

FIGURE 6. Intersection of the loops $\mathscr{L}_{32}$ and $\mathscr{L}_{100}$.

$\operatorname{arcs} \mathcal{A}_{1}$ and $\mathcal{A}_{2}$ into two pieces:

$$
\begin{array}{ll}
\mathcal{Q}_{1}=\left\{t_{1, b}, t_{1, m}, t_{2, b}, t_{2, m}\right\}, & \mathcal{Q}_{2}=\left\{t_{1, b}, t_{1, m}, t_{2, m}, t_{2, e}\right\}, \\
\mathcal{Q}_{3}=\left\{t_{1, m}, t_{1, e}, t_{2, b}, t_{2, m}\right\}, & \mathcal{Q}_{4}=\left\{t_{1, m}, t_{1, e}, t_{2, m}, t_{2, e}\right\},
\end{array}
$$

where

$$
t_{1, m}=\frac{1}{2}\left(t_{1, b}+t_{1, e}\right) \quad \text { and } \quad t_{2, m}=\frac{1}{2}\left(t_{2, b}+t_{2, e}\right) .
$$

(ii) For each subquad $\mathcal{Q}_{j}$, a crude (and fast) test was used to determine whether the $\operatorname{arcs} \mathcal{A}_{1, j}$ and $\mathcal{A}_{2, j}$ represented by $\mathcal{Q}_{j}$ were close enough that an intersection might be possible; if not, the subquad was eliminated from further consideration. Although this particular test allowed for false positives, it proved to be fairly effective in practice.

(iii) All of the subquads that survived step (ii) were collected into a new list for processing during the next cycle.

In principle, at the end of this twenty-cycle procedure, every remaining quad would give rise to a point of intersection for the loops $\mathscr{L}_{m}$ and $\mathscr{L}_{n}$. Our verification procedure can be summarized as follows:

(i) For each quad $\mathcal{Q}$ we considered not only the $\operatorname{arcs} \mathcal{A}_{1}$ and $\mathcal{A}_{2}$ defined by (7-1) but also the line segments connecting the endpoints of the arcs, namely,

$$
\mathcal{L}_{1}=\left\{L_{1}(t): t_{1, b}<t<t_{1, e}\right\}
$$

and

$$
\mathcal{L}_{2}=\left\{L_{2}(t): t_{2, b}<t<t_{2, e}\right\},
$$

where

$L_{j}(t)=\zeta\left(1 / 2+i t_{j, b}\right) \frac{t-t_{j, e}}{t_{j, b}-t_{j, e}}+\zeta\left(1 / 2+i t_{j, e}\right) \frac{t_{j, b}-t}{t_{j, b}-t_{j, e}}$

for $j=1,2$.

(ii) To find an intersection of $\mathcal{A}_{1}$ and $\mathcal{A}_{2}$, we first found numbers $t_{1}^{*}$ and $t_{2}^{*}$ such that $L_{1}\left(t_{1}^{*}\right)=L_{2}\left(t_{2}^{*}\right)$; these numbers became our initial "guess" in using the command FindRoot to locate an intersection between $\mathcal{A}_{1}$ and $\mathcal{A}_{2}$. If FindRoot returned values $t_{1}, t_{2}$ such that $t_{1} \notin\left(t_{1, b}, t_{1, e}\right)$ or $t_{2} \notin\left(t_{1, b}, t_{1, e}\right)$, then this step of the algorithm returned FAIL (in this case, the quad was not eliminated, but instead it was subjected to more iterations of the cycling process described earlier); otherwise, the algorithm proceeded to step (iii).

(iii) In this step, a special routine was used to eliminate the possibility of multiple intersections between the arcs $\mathcal{A}_{1}$ and $\mathcal{A}_{2}$. When a multiple intersection was deemed possible, this step of the algorithm returned FAIL (and as before, this quad would then be subjected to further iterations of the cycling process).

In this manner, every quad that did not fail the verification procedure gave rise to a pair of numbers $t_{1}, t_{2}$ for which $\zeta\left(1 / 2+t_{1}\right)=\zeta\left(1 / 2+i t_{2}\right) \neq 0$.

\begin{tabular}{|c|c|c|}
\hline$t_{1}$ & $t_{2}$ & $\zeta\left(1 / 2+i t_{1}\right)$ and $\zeta\left(1 / 2+i t_{2}\right)$ \\
\hline \hline $280.80242937 \ldots$ & $-280.80242937 \ldots$ & $7.00315163 \ldots$ \\
$282.45472082 \ldots$ & $-282.45472082 \ldots$ & $280.80242937 \ldots$ \\
\hline
\end{tabular}

TABLE 5. Intersections of the loops $\mathscr{L}_{126}$ and $\mathscr{L}_{-126}$. 
The same techniques were used to find selfintersections, but the initial quad was defined in a slightly different way before the cycling process began.

\section{CONCLUDING REMARKS}

Using analytic properties of $\vartheta(t)=\arg \zeta(1 / 2+i t)$, it should be possible to prove that Conjecture 1.1 holds with only countably many exceptions.

Let $\mathcal{R}=\{\sigma+i t:|\sigma-1 / 2| \leqslant \delta, 1 \leqslant t \leqslant T\}$. It is shown in [Levinson 75] that the number of solutions in $\mathcal{R}$ to the equation $\zeta(\sigma+i t)=z$ is equal to $(T / 2 \pi) \log T+O_{\delta}(T)$, whereas the number of solutions with $|\sigma-1 / 2|>\delta$ is $O_{\delta}(T)$. The latter result can be improved if and only if $z=0$, which shows that the clustering of the zeros of $\zeta(s)$ near the critical line is more pronounced than the clustering of $z$-values for any $z \neq 0$. This can be viewed as weak evidence for the conjecture (1-1), which asserts that the equation $\zeta(1 / 2+i t)=z$ has infinitely many solutions if and only if $z=0$.

It would be interesting to see whether our numerical investigation could be performed on a much larger scale to obtain more compelling evidence in support of our conjectures. We leave this project to the interested reader!

\section{REFERENCES}

[Borwein et al. 08] P. Borwein, S. Choi, B. Rooney, and A. Weirathmueller, editors. The Riemann Hypothesis: A resource for the Afficionado and Virtuoso Alike, CMS Books in Mathematics. New York: Springer, 2008.

[Conrey et al. 84] J. B. Conrey, A. Ghosh, and S. M. Gonek. "A Note on Gaps between Zeros of the Zeta Function." Bull. London Math. Soc. 16:4 (1984), 421-424.

[Edwards 74] H. M. Edwards. Riemann's Zeta Function, Pure and Applied Mathematics 58. New York: Academic Press, 1974.

[Gourdon and Demichel 04] X. Gourdon and P. Demichel. "The $10^{13}$ First Zeros of the Riemann Zeta Function, and Zeros Computation at Very Large Height." Preprint, available at http://numbers.computation.free. fr/Constants/Miscellaneous/zetazeros1e13-1e24.pdf, 2004 .
[Gram 03] J.-P. Gram. "Sur les zéros de la fonction $\zeta(s)$ de Riemann." Acta Math. 27 (1903), 289-304.

[Hardy 14] G. H. Hardy. "Sur les zéros de la fonction $\zeta(s)$ de Riemann." C. R. Acad. Sci. Paris 158 (1914), 1012-1014.

[Ingham 90] A. E. Ingham. The Distribution of Prime Numbers (reprint of the 1932 original), Cambridge Mathematical Library. Cambridge, UK: Cambridge University Press, 1990.

[Ivić 85] A. Ivić. The Riemann Zeta-Function. New York: John Wiley \& Sons, 1985.

[Karatsuba and Voronin 92] A. A. Karatsuba and S. M. Voronin. The Riemann Zeta-Function, translated from the Russian by Neal Koblitz, de Gruyter Expositions in Mathematics 5. Berlin: Walter de Gruyter \& Co., 1992.

[Levinson 75] N. Levinson. "Almost All Roots of $\zeta(s)=a$ Are Arbitrarily Close to $\sigma=1 / 2$." Proc. Nat. Acad. Sci. U.S.A. 72 (1975), 1322-1324.

[Montgomery 73] H. L. Montgomery. "The Pair Correlation of Zeros of the Zeta Function." In Analytic Number Theory: Proc. Sympos. Pure Math., Vol. XXIV, St. Louis Univ., St. Louis, Mo., 1972, pp. 181-193. Providence: Amer. Math. Soc., 1973.

[Montgomery and Vaughan 07] H. L. Montgomery and R. C. Vaughan. Multiplicative Number Theory I. Classical Theory, Cambridge Studies in Advanced Mathematics 97. Cambridge, UK: Cambridge University Press, 2007.

[Patterson 88] S. J. Patterson. An Introduction to the Theory of the Riemann Zeta-Function, Cambridge Studies in Advanced Mathematics 14. Cambridge, UK: Cambridge University Press, 1988.

[Riemann 59] B. Riemann. "Ueber die Anzahl der Primzahlen unter einer gegebenen Grösse." Monatsberichte der Berliner Akademie, 1859.

[Selberg 92] A. Selberg. "Old and New Conjectures and Results about a Class of Dirichlet Series." In Proceedings of the Amalfi Conference on Analytic Number Theory (Maiori, 1989), pp. 367-385. Univ. Salerno, 1992.

[Titchmarsh 86] E. C. Titchmarsh. The Theory of the Riemann Zeta-Function, second edition. Oxford: Oxford University Press, 1986.

William D. Banks, Dept. of Mathematics, University of Missouri, Columbia, MO 65211, USA (bankswd@missouri.edu)

Sarah Kang, Dept. of Mathematics, University of Missouri, Columbia, MO 65211, USA (sk244@mail.missouri.edu)

Received January 20, 2011; accepted September 20, 2011. 